\title{
Activación conductual y depresión: Conceptualización, evidencia y aplicaciones en Iberoamérica
}

\section{Behavioral activation and depression: Conceptualization, evidence and applications in Latin America}

\author{
Javier Bianchi \\ Ángela Henao \\ Fundación Universitaria Konrad Lorenz, Bogotá, Colombia
}

(Rec: 28 agosto 2013 / Acept: 12 Mayo 2015)

\begin{abstract}
Resumen
La depresión es uno de los problemas en salud mental más prevalentes. En Latinoamérica se constituye como la primera problemática en cuanto a años de vida perdidos por incapacidad, lo que implica que se deben hacer esfuerzos importantes en desarrollar estrategias de intervención eficaces para su intervención. Este artículo tiene como objetivo, en primer lugar, ofrecer una revisión conceptual de la activación conductual, una de las estrategias de intervención que han probado ser eficaces en el tratamiento de la depresión y sus diferentes modalidades, en segundo lugar, examinar el uso que ha tenido en el manejo de la depresión en diferentes poblaciones y por último, revisar la evidencia empírica en Iberoamérica.

Palabras clave: depresión, activación conductual, evidencia empírica e Iberoamérica
\end{abstract}

\begin{abstract}
Depression is nowadays one of the most prevalent mental health problems. In Latin America is the main problem in terms of years of life lost due to disability, implying that major efforts should be made to develop effective intervention strategies for treatment. This article aims, first, to provide a conceptual review of the Behavioral Activation, one of the interventions that have proven to be effective to treat depression and its various forms, secondly, to examine its use to treating depression with different populations and finally, to review the empirical evidence in Latin America.

Keywords: depression, behavioral activation, empirical evidence, Iberoamerica
\end{abstract}

\footnotetext{
Correspondencia: Javier M. Bianchi, Facultad de Psicología, Fundación Universitaria Konrad Lorenz, Bogotá, Colombia, dirección de contacto: javierm. bianchis@konradlorenz.edu.co y biancosal@gmail.com; Ángela M. Henao, Facultad de Psicología, Fundación Universitaria Konrad Lorenz, Bogotá, Colombia, dirección de contacto: angelam.henaog@konradlorenz.edu

Nota: Este artículo se derivó de la investigación: Efecto del tratamiento de Activación Conductual (BATD-R) sobre conductas objetivo y estimación verbal del estado de ánimo: Un diseño intrasujeto. Fue financiada por la Fundación Universitaria Konrad Lorenz y desarrollada en la línea de investigación en conceptualización, evaluación e intervención en psicología clínica del grupo de investigación en ciencias del comportamiento.
} 


\section{Introducción}

La depresión es considerada el resfriado común de los trastornos psiquiátricos (Aguado, Silberman y Manrique, 2005), debido a sus altas tasas de incidencia y prevalencia. De acuerdo con la Organización Mundial de la Salud, ocupa el tercer lugar dentro de las causas de discapacidad, antecedida por pérdida auditiva y errores refractivos (WHO, 2008). Gotlib y Hammen (2009) refieren que un estimado del 20\% de la población norteamericana experimenta un episodio clínico de depresión alguna vez en la vida, principalmente las mujeres. Alrededor del $20 \%$ de los adultos y del $50 \%$ de niños y adolescentes expresan síntomas depresivos en lapsos entre 1 semana y 6 meses (Kessler y Wang, 2009).

En Latinoamérica, el estudio realizado por Khon et al. (2005) sobre la prevalencia de trastornos mentales en América Latina y el Caribe, en el que recopilaron datos de estudios comunitarios publicados entre 1980 y 2004 , establece una tasa de prevalencia de $4.9 \%$ para la depresión mayor, siendo la segunda problemática con mayor prevalencia, después de la dependencia de alcohol con un 5.7\%. Ortiz, López y Borges (2007) y Roses (2005) reportan que los trastornos depresivos unipolares ocupan la primera posición en América en las problemáticas de salud mental, que ocasionan mayores años de vida perdidos por discapacidad con un $8 \%$. En México, la Encuesta Nacional de Epidemiología Psiquiátrica informa que el $9.2 \%$ de personas sufren un trastorno afectivo en algún momento de su vida y el $4.2 \%$ durante el último año (Berenzon, Lara, Robles y Medina, 2013); en Uruguay, en el estudio epidemiológico realizado por Díaz y Ruiz (2002) en el Hospital de Clínicas, se encuentra que de 864 pacientes estudiados, el $26.3 \%$ presentaba depresión mayor; por su parte, Minoletti y Zaccaria (2005) en Chile mencionan una prevalencia de $9.0 \%$ para la depresión constituyéndose en la segunda problemática más prevalente en ese país.

En Colombia, el estudio de salud mental realizado en 2003 por el Ministerio de Protección Social establece una prevalencia de $15 \%$ para los trastornos del estado de ánimo, correspondiente al segundo trastorno más frecuente en la población (Posada-Villa, Aguilar-Gaxiola, Magaña y Gómez, 2004). De otra parte, Gómez, Bohórquez, Pinto, Gil, Rondón y Díaz-Granados (2004), a través de una encuesta aplicada a 1116 adultos con el fin de establecer la prevalencia de la depresión durante el periodo 2000-2001, encontraron que el $10 \%$ de la muestra reportaba presencia de algún episodio depresivo durante los últimos 12 meses previos a la encuesta y el $8.5 \%$ presentaron algún episodio durante el último mes. Más recientemente, en 2012, algunos estudios (Castro, Escobar, Sáenz, Delgado, et al., 2012; Díaz, 2012; López,
Montoya y Dussán, 2012) que la depresión es el problema con mayor frecuencia reportado en los centros de atención en salud mental.

Debido a estas altas tasas de prevalencia de la depresión, es necesario contar con estrategias de intervención que sean eficaces para atender este tipo de problemáticas. Dentro de las alternativas de intervención psicológica que han mostrado mayor evidencia empírica se encuentra la terapia cognitivo conductual (TCC), cuyo objetivo principal es la modificación de los pensamientos distorsionados que se consideran la causa de la problemática; esta terapia cuenta con varios estudios publicados que muestran su grado de eficacia (Hollon y Ponniah, 2010; DeRubeis et al., 2005; Butler, Chapman, Forman y Beck, 2006).

Por otro lado, dentro de las terapias de tercera generación, también denominadas terapias contextuales, que se alejan de la tradición cognoscitiva y se aproximan a los planteamientos del conductismo radical, haciendo énfasis en el rol que tiene el contexto y el análisis idiográfico de las problemáticas (Pérez, 2012), la Activación Conductual (AC), es una de las alternativas más utilizadas en el tratamiento de la depresión, ya que es un tratamiento estructurado, eficaz y bien establecido (Puspitasari, Kanter, Koerner, Murphy y Crowe, 2013; Martell, Dimidjian y Dunn, 2010) que está dirigido al incremento, de manera estructurada, de las conductas manifiestas que pueden acceder a contingencias medioambientales reforzantes y subsecuentemente mejorar el estado de ánimo y la calidad de vida (Hopko et al., 2011; Hopko, Lejuez, Ruggiero y Eifert, 2003); a diferencia de la terapia cognitiva, la $A C$ busca cambiar las interacciones persona-ambiente, las cuales resultarán en subsecuentes cambios internos (Ekers, 2011; Pérez, 2007).

Es a partir del estudio de desmantelamiento de la TCC de Jacobson et al. (1996), en el que se demuestra que el componente de Programación de Actividades es igualmente eficaz como el tratamiento multicomponente completo, que resurge el interés en la Programación de Actividades $/ A C$ como un tratamiento en sí mismo para la depresión.

\section{Propuestas de activación conductual}

Este interés por las intervenciones fundamentadas en la $A C$ conlleva el desarrollo de tres vertientes distintas; la primera de ellas nace del equipo de Jacobson et al. (1996) y de su estudio, propuesta por Martell et al. (2010), la cual se consolida en la actualidad como una de las opciones de intervención para la depresión. Este modelo de $A C$ es una intervención idiográfica y analítica funcional, que se fundamenta en los aspectos funcionales de la conducta depresiva 
(Ekers, 2011). Por medio de la $A C$ se identifican patrones evitativos que pueden estar manteniendo la depresión y promueve incrementar actividades placenteras o productivas que pueden proveer refuerzo positivo, por ende mantenerse, con la subsecuente mejora en el estado de ánimo (Bottonari, Roberts, Thomas y Read, 2008; Dimidjian, Martell, Addis y Herman-Dunn, 2008; Martell et al., 2010). En este sentido, la $A C$ no está dirigida a incrementar acceso a fuentes de placer o a introducir actividades que mejoren el estado de ánimo; está dirigida a incrementar las actividades que le permitan acceder a fuentes de recompensa en la vida de las personas, así como solucionar problemas; por lo tanto, aquellas actividades de evitación y escape, que se mantienen por refuerzo negativo, son antagónicas con los objetivos de activación (Dimidjian et al., 2008; Cullen, Spates, Pagoto y Doran, 2006).

La $A C$ que nace del estudio de Jacobson et al. (1996), publicó una primera guía denominada Depression in Context: Strategies for Guided Action (Martell, Addis y Jacobson, 2001), que estaba dirigida a fundamentar desde una perspectiva contextual las técnicas de $A C$. Dentro de esta primera corriente de $A C$ como terapia de tercera ola, la más reciente guía publicada corresponde a Behavioral Activation for Depression: A Clinician's Guide (Martell et al., 2010).

Por otro lado, la segunda vertiente o propuesta de $A C$ corresponde al Tratamiento Breve de Activación Conductual para Depresión (BATD, por sus siglas en inglés). Uno de los pilares básicos que sustentan esta versión de $A C$ corresponde a la ley de igualación de Herrnstein de 1970 (Hopko et al., 2003; Lejuez, Hopko, LePage, Hopko y McNeil 2001; LeJuez, Hopko, Acierno, Daughters y Pagoto, 2010). Esta aproximación explica cómo operan los organismos (tanto animales como humanos) en situaciones donde no existe una única alternativa de respuesta, la cual se ve reducida en explicaciones desde programas de reforzamiento simple (Caycedo, Sandoval, Gutiérrez y Pereira, 1994). En términos de lo que se considera depresión, lo que sugieren los autores al aplicar la ley de igualación es que el tiempo y esfuerzo asignado a realizar conductas depresivas en relación con conductas no depresivas es directamente proporcional al valor del refuerzo recibido al emitir este tipo de comportamientos depresivos frente a los no depresivos (Hopko et al., 2003). En otras palabras, cuando el valor del refuerzo de conductas depresivas se incrementa, se decrementa el valor del refuerzo de las conductas no depresivas o saludables; por lo tanto, al recibir mayor refuerzo las conductas no saludables o depresivas, éstas incrementarán la posibilidad de mantenerse en el tiempo (Hopko et al., 2003; Kanter, Busch,
Weeks y Landes, 2008). Esto implica que la reducción de comportamientos depresivos no es el simple resultado de la disminución de refuerzo positivo, sino que se debe a las tasas o proporciones de refuerzo de las conductas depresivas con respecto a las conductas no depresivas (Kanter, Busch, Weeks y Landes, 2008), lo que conlleva que en la evaluación es necesaria una mayor búsqueda de variables contextuales en las cuales la conducta ocurre, no sólo un reforzador que sigue a una conducta específica (Kanter, Busch y Rusch, 2009); es decir, la suma total del refuerzo disponible en el ambiente de una persona debe ser tenido en cuenta, no concentrarse exclusivamente en las conductas blanco y sus consecuencias (Kanter et al., 2008).

El modelo BATD publicó su primer manual de tratamiento denominado A Brief Behavioral Activation Treatment for Depression (LeJuez, Hopko, LePage, Hopko y McNeil, 2001); su versión más reciente corresponde a Ten Year Revision of the Brief Behavioral Activation Treatment for Depression: Revised Treatment Manual (BATD-r).

Por último, la $A C$ propuesta por Kanter et al. (2009) se configura como una nueva forma de activación. Esta versión más reciente del tratamiento comparte los fundamentos y técnicas propuestas en los otros dos modelos de $A C$; no obstante, estos autores estructuran el tratamiento de manera tal que pretenden optimizar las estrategias propias de la activación iniciando lo que denominan Activación Simple, la cual se fundamenta en la estructuración de actividades encaminadas hacia el valor. Estas sesiones iniciales incluyen monitoreo de actividades, evaluación de valores y planeación de actividades encaminadas al valor. Si esta activación simple no resulta, se procede a realizar una evaluación funcional, la cual determinará cuáles son los focos principales de intervención en la persona en particular, conllevando a una individualización de la propuesta y del plan de intervención (Kanter et al., 2009).

En este apartado se revisaron las diferentes propuestas de $A C$ (el modelo de Martell et al.. 2001, 2010; el modelo de Hopko et al., 2001, 2010; y la propuesta de Kanter et al., 2009). Además, se mencionaron las diferentes guías de intervención clínica que cuentan con la programación de actividades como su componente principal. A continuación se revisará la evidencia empírica de la $A C$ como un procedimiento eficaz para la depresión.

\section{Evidencia empírica de la activación conductual}

En relación con la evidencia empírica de este tratamiento se cuenta con diversos estudios que demuestran su efectividad en la disminución de los síntomas depresivos, demostrando 
que la $A C$ goza de buena reputación en el campo clínico y que ha demostrado su apoyo empírico. Entre estos estudios se puede mencionar el realizado por Cullen et al. (2006), con personas que tenían un diagnóstico de trastorno depresivo mayor, en el que se compara el grupo de tratamiento con $A C$ con un grupo control de lista de espera, encontrando que la $A C$ logra reducciones significativas en los puntajes de depresión. En Suecia, Freij y Masri (2008) implementaron la $A C$ con ocho adultos que presentaban depresión unipolar, obteniendo una disminución de los síntomas depresivos y una mejora en el nivel de funcionamiento. En Australia, Bailey y Arco (2010) aplicaron un tratamiento de $A C$ en dos adultos con síntomas moderados de depresión y lograron el decremento de los síntomas de la depresión y un incremento de actividades de diferentes niveles de dificultad.

En Estados Unidos, Gawrysiak et al. (2012) reportan un estudio de caso en una paciente de 64 años, diagnosticada con cáncer de mama 4 meses antes de la evaluación pretratamiento; adicionalmente presenta un cuadro depresivo y de ansiedad generalizada, el cual se exacerba posterior al diagnóstico de cáncer. Se aplicó el protocolo BATD en un total de 8 sesiones con una duración de 1 hora por sesión. Las medidas fueron tanto psicológicas (escalas como Inventario de Beck para Depresión II y Escala de Observación de Recompensa Ambiental), como de neuroimagen, Resonancia Magnética Funcional (fMRI). Los resultados encontrados muestran una mejora clínica en la depresión de la paciente, la cual se apoya en los cambios observados en el nivel de dependencia de oxigenación sanguíneo en la corteza prefrontal y en la corteza cingulada, de acuerdo con la fMRI. Estos resultados muestran tanto los cambios en los mecanismos neurobiológicos implicados en la psicopatología depresiva luego de la aplicación del BATD, como la eficacia de la psicoterapia.

En relación a la población latina, en Norteamérica hay tres estudios significativos. El primero de ellos corresponde a la activación conductual como un enfoque de tratamiento para latinos con depresión (Santiago-Rivera et al., 2008). En este estudio se realiza una contextualización sobre la adaptación de la activación conductual a las características culturales propias de la población latina en Norteamérica. En la adaptación a latinos tuvieron en cuenta los propósitos de activación sensibles a la cultura, centrarse en los eventos estresantes y la evitación, abordar el compromiso con el tratamiento, e incorporar los recursos familiares, sociales y comunitarios. Adicionalmente, se reporta un estudio de caso con resultados satisfactorios y promisorios para esta adaptación. El segundo estudio de la literatura revisada corresponde a la publicación de Kanter, Diéguez, Rusch,
Busch y Santiago-Rivera (2008), un estudio de caso único con una mujer de 25 años con depresión, y encontraron que la aplicación de la adaptación de la $A C$ para latinos fue exitosa. Por último, Kanter, Santiago-Rivera, Rusch, Busch y West (2010) realizaron un estudio en el que adaptaron un protocolo de $A C$ para latinos, con el objetivo de evaluar la adaptación del tratamiento en población latina que presentaba depresión, debido a los múltiples factores estresores ambientales a los que se ve sometida esta población en Estados Unidos como inmigrantes. Los investigadores encontraron que este tratamiento apoyaba la facilidad y efectividad de la $A C$ en este tipo de poblaciones.

Por otro lado, se encuentran pocos reportes de investigación en relación con el uso de la $A C$ en población adolescente. Al respecto, Ruggiero, Morris, Hopko y LeJuez (2007) publican un estudio de caso con una adolescente de 17 años. Los resultados fueron satisfactorios con un incremento en las tasas de reforzamiento y un decremento en los síntomas depresivos. El tratamiento se desarrolló en 8 sesiones, lo cual repercute en que los autores sugieran que BATD puede ser aplicado de manera exitosa con adolescentes al igual que con adultos. En tanto, Jacob, Keeley, Ritschel y Craighead (2011) realizaron un estudio con tres adolescentes afroamericanos que cumplían criterios para trastorno depresivo mayor. Los resultados arrojaron que dos de los tres participantes después de la aplicación del tratamiento de $A C$ ya no cumplían criterios para depresión mayor. Se halló adicionalmente que los participantes lograron entender y aplicar las razones del tratamiento en su vida diaria.

En cuanto a estudios que evalúan y clasifican investigaciones que brindan evidencia de la $A C$, podemos mencionar la investigación de Spates, Pagoto y Kalata (2006), la que concluye que este tratamiento tiene soporte empírico para el tratamiento de la depresión. Además, el meta-análisis de Cuijpers, Van Straten y Warmerdam (2007) concluye igualmente que el tratamiento de $A C$ es eficiente para tratar la depresión y además sencillo debido a que no requiere habilidades complejas para su aplicación. Por otro lado, el meta-análisis realizado por Mazzucchelli, Kane y Rees (2009), en el que se efectúa la revisión de 34 artículos publicados entre 1970 y 2008 sobre $A C$, concluyó que era efectiva para tratar la depresión y que podría considerarse como una "intervención bien establecida y probablemente eficaz” (p. 383). Por último, el meta-análisis de Ekers, Richards y Gilbody (2008), en el que se analizaron 17 estudios, establece que al comparar la $A C$ con grupos control, psicoterapia breve y terapia de apoyo, ésta era más efectiva, y al compararla con la TCC era igualmente efectiva. 


\section{Activación conductual y problemas clínicos}

La $A C$ ha sido empleada también en el tratamiento de personas que presentan otros problemas clínicos en comorbilidad con la depresión. Entre ellos se encuentra el estudio Romero, Oñate y Vucinovich (2011), en el que presentan resultados positivos en la disminución de síntomas depresivos y síntomas psicóticos, así como un incremento en los niveles de reforzamiento positivo percibido, utilizando la $A C$ en una persona con esquizofrenia.

El estudio de caso de Hopko, Lejuez y Hopko (2004) demuestra la eficacia de la $A C$ en el tratamiento de conductas de ansiedad comórbidas con síntomas depresivos. Los investigadores confirman que después de un tratamiento de 10 sesiones, el consultante presenta un decremento en la ansiedad y la depresión reportada y un incremento en su calidad de vida.

Magidson et al. (2011) evaluaron la efectividad de un tratamiento que incluía un componente de $A C$ en 58 adultos que tenían abuso de sustancias y lo compararon con un tratamiento de apoyo. Al final de la intervención ambos grupos presentaron disminución en el nivel de severidad de depresión, sin embargo, el grupo que fue tratado con el componente de $A C$ adicionalmente presentó tasas más altas de permanencia en el tratamiento para el abuso de sustancias y mayor activación según la Escala de Depresión de Activación Conductual.

En el área de salud, específicamente oncología, hay diferentes estudios que arrojan resultados positivos en la mejoría de los pacientes posterior a la implementación de la $A C$, entre éstos está el de Hopko, Armento, Hunt, Bell y Lejuez (2005), quienes hicieron un estudio con seis pacientes de un hospital que tenían cáncer y síntomas de depresión. Aquí se utilizó un protocolo breve de $A C$, el cual fue efectivo para la reducción de síntomas depresivos, así como en el incremento de la satisfacción por parte de los pacientes con el tratamiento y un mantenimiento de los resultados tres meses después de terminado el tratamiento. Posteriormente, Hopko et al. (2011) comparan la aplicación de un tratamiento de $A C$ con un tratamiento de solución de problemas a un grupo de mujeres con cáncer y depresión mayor, hallando que ambos tratamientos eran eficaces en la reducción de síntomas depresivos y en el mejoramiento de la calidad de vida.

\section{Variantes de la intervención con activación conductual}

En Tennessee, Gawrysiak, Nicholas y Hopko (2009) realizaron un estudio con población no clínica con 30 estudiantes universitarios que presentaban síntomas depresivos. La variante interesante de esta investigación es que el tratamiento propuesto consistía en una única sesión en formato individual. La sesión de intervención tenía una duración aproximada de 90 minutos, en la cual se establecían conductas orientadas hacia valor, las cuales se tenían que implementar en un intervalo de dos semanas. Posterior a la aplicación del tratamiento BATD se encontró mayor reducción en los síntomas de depresión comparados con el grupo control y mayor recompensa ambiental en el posttratamiento, lo que se asoció con mayor tamaño del efecto (Gawrysiak et al., 2009).

Otra variante interesante del BATD se implementó en una muestra de 50 participantes, a quienes se les aplicó un tratamiento fundamentado en Activación Conductual de Comportamientos Religiosos (BARB, en sus siglas en inglés) de una sola sesión, en contraste con una terapia de apoyo (Armento, McNulty y Hopko, 2012). Los resultados mostraron que en comparación con el grupo de terapia de apoyo, el grupo que recibió el BARB reportaron cambios significativos en el post-tratamiento, tanto en sus conductas religiosas como en sus actitudes y estrategias de afrontamiento. Adicionalmente, hubo disminución en los síntomas de ansiedad y de depresión, así como un incremento en la calidad de vida (Armento et al., 2012).

MacPherson et al. (2010), trabajaron con 68 fumadores regulares que consumían 10 o más cigarrillos diarios; adicionalmente presentaban puntuaciones iguales o superiores a 10 en el Inventario de Beck para Depresión II. Se compararon dos intervenciones, el Tratamiento de Activación Conductual para Fumadores (BATS, en sus siglas en inglés), una variante del BATD, y el tratamiento estándar; el formato de tratamiento fue grupal. Los resultados del estudio muestran que el BATS presenta una mayor probabilidad de abstinencia en los seis meses de seguimiento entre los fumadores con elevados síntomas depresivos; además, los síntomas depresivos fueron más bajos durante el seguimiento para el grupo BATS que para el estándar. Por lo tanto, el BATS parece ser una intervención prometedora para este tipo de problemáticas y 
por su extensión corta podría ser una buena alternativa en diferentes escenarios y poblaciones con problemas vinculados con tabaquismo (MacPherson et al., 2010).

Dentro de las variantes que no son específicamente del tratamiento, se encuentra en la literatura el entrenamiento realizado a no especialistas para la administración de la $A C$. Ekers et al. (2011) reportan el estudio efectuado con 47 participantes, los cuales estaban siendo atendidos en servicios de salud se encontraban diagnosticados con depresión. El tratamiento fue administrado por enfermeras en salud mental, quienes recibieron entrenamiento durante cinco días y supervisión quincenal por el investigador principal. Los resultados muestran una buena respuesta en el tratamiento de $A C$ administrado por no especialistas, lo cual tiene implicaciones económicas importante para el servicio de salud, como para la diseminación de la $A C$.

Recientemente, Puspitasari et al. (2013) realizaron dos estudios secuenciales dirigidos a revisar el entrenamiento de proveedores de salud mentalcomunitarios en $A C$. Lo interesante de este estudio es la modalidad de entrenamiento el cual fue fundamentado en el aprendizaje activo, modular y on-line. Este tipo de herramientas on-line de manera modular, redunda en un alto grado de satisfacción en quienes recibieron el entrenamiento y una retroalimentación de la mejora en habilidades clínicas en la implementación de la $A C$.

El uso de la $A C$ en problemáticas relacionadas con comportamientos depresivos, que no necesariamente se circunscriben a miradas nosológicas o taxonómicas mutuamente excluyentes como lo sería el diagnóstico de depresión, ha tenido una gran proliferación, lo que se evidencia en los diferentes estudios encontrados y referidos en este apartado. A continuación se nombrarán algunos de los reportes hallados en Iberoamérica, que es uno de los objetivos de este artículo.

\section{Usos en Iberoamérica}

El impacto en Iberoamérica de la $A C$ ha sido favorable tanto para los programas de formación de psicólogos como para la revisión teórica, lo cual ha permitido la formación de profesionales en psicología en estrategias propias de esta terapia de tercera ola, que ha mostrado una corta historia pero un pasado extenso. Esta afirmación se puede medir en diferentes aspectos, tales como los módulos de formación en programas de postgrado en psicología clínica, las publicaciones de revisión y de investigación, las capacitaciones de profesionales por parte de autores de este tipo de intervenciones (v.gr. taller dictado por Kanter en 2012, en
Bogotá, Colombia), la traducción de protocolos (v.gr. BATD, traducida por el grupo ACT en Argentina), entre otros.

En España es donde quizá se encuentra mayor información en relación con la $A C$. Los dos autores más representativos que han trabajado en $A C$, como Terapias de Tercera Generación o de la Tercera Ola, son Jorge Barraca y Marino Pérez (entre muchos otros, tales como Carmen Luciano con la ACT). Estos autores tienen varias publicaciones relacionadas con estas terapias de tercera generación, dentro de las cuales podemos hablar de libros como La psicoterapia desde el punto de vista del conductista (Pérez, 1996), o La Mente O La Vida: Una aproximación a la Terapia de Aceptación y Compromiso (Barraca, 2005); artículos de revisión sobre la tercera ola y $A C$ (Barraca, 2009; Pérez, 2007; Pérez, 2008; Barraca, 2011a); adaptaciones españolas de escalas relacionadas con $A C$ (Barraca y Pérez, 2010; Barraca, Pérez y Lozano, 2011); estudios de caso (Barraca, 2010); y otro tipo de publicaciones vinculadas con $A C$ y las terapias de tercera generación. A continuación se revisarán brevemente algunas de estas publicaciones, en especial aquellas que tienen que ver específicamente con $A C$.

Dentro de los artículos de revisión publicados está el de Barraca (2011a), en el cual se revisan las terapias de la tercera ola frente a las terapia cognitivas en cuanto al control mental versus la aceptación; el autor también resalta el papel de la Activación Conductual propuesta por Martell et al. (2001) y Martell et al. (2010), quienes introducen elementos relacionados con el Mindfulness para atender la rumiación cognitiva que puede interferir con la programación de actividades, en contraposición con la propuesta de Lejuez et al. (2001) y Lejuez et al. (2010), quienes no proponen una intervención directa en lo cognitivo y asumen que desaparecerá dicha interferencia una vez mejore el estado de ánimo. Este mismo autor publica un artículo sobre la $A C$, en donde plantea la comparación de las dos formas de intervención, la de Martell et al. (2001) con la de Lejuez et al. (2001). En su artículo revisa los aspectos comunes y diferenciales de estas dos aproximaciones de la $A C$, tanto en los antecedentes históricos como en sus supuestos teóricos, en el trabajo cognitivo y la estructuración de la terapia, entre otros tópicos relevantes (Barraca, 2009). Además, publica el artículo Tratamiento de Activación Conductual Para La Depresión (TACD): Descripción, técnicas y aplicación, donde realiza nuevamente un barrido por los principios, formatos de intervención y evidencia empírica de la $A C$ (Barraca, 2010). Quizá la más notable reflexión sugerida por este autor, dentro de muchas otras, con relación al BATD es "Si la evidencia experimental indica que un terapeuta puede 
ser eficaz siguiendo un enfoque tan parsimonioso, ¿por qué complicar más la intervención?" (Barraca, 2010, pp. 38).

Por su parte, Marino Pérez (2007) publica un artículo titulado: La Activación Conductual y la Desmedicalización de la Depresión. En este artículo realiza una excelente revisión teórica de la propuesta de Martell et al. (2001), acompañada de las implicaciones tanto epistemológicas como prácticas de esta alternativa de tratamiento para una de las primeras causas de morbilidad en el mundo.

Adicionalmente, en España hay trabajos donde se utiliza la $A C$ en población clínica. Fernández et al. (2011) encontraron que pacientes con cáncer que presentaban síntomas depresivos, con el tratamiento de $A C$ lograban mayor recuperación de actividades cotidianas y de ocio y no presentaban alteraciones emocionales. Por otro lado, Bajona (2012) exhibe resultados positivos en una muestra de 12 pacientes que asistían a un centro de atención primaria en salud, a los que se les interviene empleando un protocolo cognitivo conductual, de formato grupal, al cual se le anexa una fase de intervención de $A C$. La evaluación post-tratamiento presenta un decremento en los puntajes del BDI y una mejora en la iniciativa para realizar actividades. De igual forma, Barraca (2011b) presenta resultados positivos en la reducción de síntomas depresivos en un hombre con depresión posterior a la implementación de esta intervención.

En México, en la búsqueda de información se encuentran dos artículos que hacen referencia a la $A C$. El primero es de Jorge Salinas (2010), que publica un reporte de caso titulado El Tratamiento de la Depresión con un Enfoque de Activación Conductual: Un Caso. En dicho reporte refiere que no parece necesaria una intervención cognitiva como la sugerida por la TCC de Beck, e informa remisión de síntomas depresivos como resultado del incremento de la actividad social y laboral del paciente; no obstante, no quedan claras las variables evaluadas. La intervención referida tiene componentes tales como relajación muscular progresiva y entrenamiento en terapia racional emotiva, lo cual no apoyaría del todo la eficacia de la $A C$ como técnica para casos de depresión, ya que al parecer implementó un tratamiento multicomponente. La intervención cognitiva propuesta por el autor no parece estar relacionada con una mirada funcional contextual. Por otro lado, Becerra, Lugo y Reynoso (2013) realizan un estudio con pacientes oncológicos hospitalizados, identifica una serie de dificultades contextuales con estados de ánimo bajo y ansiedad, que redundan en una estancia hospitalaria que afecta el estado de ánimo y que de no tratarse podría agudizarse; por tal razón, consideran oportuno para dicha población específica la implementación de la terapia de $A C$, como una alternativa posible para atender estas necesidades relacionadas no sólo con la estancia de los pacientes, sino que podrían repercutir en su condición de salud.

En Colombia se tiene información sobre dos estudios realizados en la Universidad Javeriana. El primero es un estudio de caso de (Navas, 2011), cuyo objetivo era demostrar la eficacia de la $A C$ en las conductas depresivas de 3 consultantes de un centro de atención en psicología. Posterior a la implementación del tratamiento se evidencia una disminución en las conductas depresivas y un aumento en las actividades agradables. El segundo estudio corresponde al realizado por Acuña (2011), en el que se utilizó la $A C$ en tres pacientes con enfermedad renal, con el objetivo de establecer la eficacia de esta intervención en tres conductas: actividad física, actividad recreativa y percepción de satisfacción. Posterior al tratamiento se encuentra que los pacientes presentan un incremento en las conductas objetivo.

Recientemente Bocanegra y Díaz (2013) desarrollaron una investigación con tres adolescentes que presentaban comportamientos depresivos, en un centro de psicología de una universidad bogotana; se implementó la guía BATD-r con la traducción de Collado, Castillo, Maero, LeJuez y MacPherson (2014). Los resultados mostraron un incremento en las tasas de conductas saludables y un decremento en los puntajes de la escala autoaplicada de Zung para depresión, validada por Lezama (2007).

\section{Conclusiones}

Esta revisión sobre el tratamiento de $A C$ establece que éste centra su atención en el análisis funcional de las conductas depresivas o no saludables de los consultantes y este análisis es el que permite identificar las conductas o actividades que, en el contexto particular, potencialmente incrementan el acceso a fuentes de reforzamiento positivo y decrementan el refuerzo negativo obtenido por las conductas poco saludables.

Igualmente, hace una revisión de diferentes estudios que han mostrado la eficacia que tiene en la intervención de la depresión y en algunas problemáticas asociadas como conductas de ansiedad, síntomas psicóticos, así como estudios en los que la depresión se asocia a condiciones de salud física como cáncer y otras enfermedades crónicas. Igualmente, se presentan estudios en los que se muestran resultados exitosos en problemáticas como consumo de cigarrillo.

$\mathrm{La} A C$ tiene una corta historia pero un pasado extenso. Surge desde las explicaciones conductuales pioneras de Skinner, Ferster, Lewinsohn y Rehm (Martell et al., 2001; 
Hopko et al., 2003) y se consolida como una alternativa de intervención en los trabajos de Beck, Rush, Shaw y Emery (1983), y Lewinsohn, Hoberman y Clarke (1989), dentro de un tratamiento multicomponente. El estudio de Jacobson et al. (1996) es el hito de su incursión dentro de las terapias de tercera generación, y a partir de dicho estudio se cuenta con tres tipos de $A C$ que han recaudado evidencia acerca de su utilidad y se consolida como uno de los tratamientos de elección para la depresión. Siguiendo a Marino Pérez (2007), la $A C$ dirigida al cambio de la relación que uno mantiene con la "situación depresógena" y que a su vez lo mantiene a uno deprimido, resulta la terapia más eficaz para la depresión. Dentro de sus características más importantes está ser una alternativa de fácil entrenamiento y cuenta con protocolos de intervención de corta duración, incluyendo los estudios de única sesión referenciados para población no clínica.

Acerca del trabajo con latinos que viven en Norteamérica, las tres publicaciones sobre el uso exclusivo de la $A C$ para el tratamiento de la depresión sugieren tener en cuenta aspectos tales como: a) la conceptualización de caso debe fundamentarse en la flexibilidad de los protocolos de activación enfocados a blancos de tratamiento así como variables culturales; b) de acuerdo a la evaluación de cada caso, factores tales como el familismo deben considerarse a la hora de instaurar una estrategia de intervención en $A C$; c) en algunos casos es necesario revisar la planeación de actividades que no necesariamente estén vinculadas con la conceptualización de caso, en especial en situaciones donde la pasividad esté lo suficientemente generalizada (Santiago-Rivera et al., 2008; Kanter et al., 2009; Kanter et al., 2010). En los tres estudios los resultados son promisorios para la adaptación de la $A C$ en latinos (BAL), aunque falta investigación para mayor apoyo empírico en este tipo de población.

Los tratamientos de una sola sesión han incursionado en las dinámicas de intervención en poblaciones no clínicas. Es el caso de los estudios en BATD (Gawrysiak et al., 2009; Armento et al. 2012), que han diseñado intervenciones de una única sesión de $A C$ con resultados promisorios. Asímismo, los resultados de las variaciones de tratamiento de $A C$, en especial del protocolo BATD (BATS, BARB, LETS ACT), han mostrado que posiblemente la planeación de actividades no sólo sea el componente activo del tratamiento de la TCC para la depresión (Jacobson et al., 1996), sino que tiene un amplio espectro de posibilidades terapéuticas que no se reducen a lo que nosológicamente se ha circunscrito dentro de la categoría nomotética de depresión. Estas variaciones, tanto en la adecuación de la $A C$ a poblaciones y problemáticas distintas como en la duración de la intervención, abren un panorama promisorio para la versatilidad, aplicabilidad, parsimonia y entrenamiento de esta intervención que cuenta con apoyo empírico y que podría ajustarse a las restricciones tanto en tiempo como en acceso a servicios adecuados de atención a problemáticas de salud que van en crecimiento a nivel mundial y que se asocian con altas tasas de prevalencia, incidencia, ausentismo laboral, calidad de vida y discapacidad.

El trabajo en población adolescente cuenta con apoyo empírico en tres estudios encontrados en la búsqueda literaria (Ruggiero et al., 2007; Jacob et al., 2011; Bocanegra y Díaz, 2013). Los resultados son promisorios para la implementación de la $A C$ en esta población en particular, aunque los autores sugieren contemplar cierto tipo de variables, tanto culturales como propias de la adolescencia a la hora de implementarla.

En Iberoamérica existen pocas publicaciones e investigaciones relacionadas con la $A C$; prevalecen los trabajos en España tanto de revisión de las terapias de tercera ola incluida la $A C$, como investigaciones encaminadas a apoyar medidas propias de la $A C$ e intervención de casos (Barraca, 2009; Pérez, 2007; Pérez, 2008; Barraca, 2011b; Barraca y Pérez, 2010; Barraca et al., 2011; Barraca, 2010; Fernández et al., 2011; Bajona, 2012). En México se hallaron dos publicaciones relacionadas con la $A C$. En una se realiza la aplicación de la $A C$, aunque no parece clara su conceptualización ni su implementación; en la otra se sugiere su aplicación en población hospitalaria pero no hay reporte de intervención (Salinas, 2010; Becerra et al., 2013). En Argentina se encuentra la traducción de la guía BATD-r por Maero et al. (2013). Por último, en Colombia se encuentran 3 tesis de grado relacionadas con estudios de caso, dos de ellas en población adulta y una con adolescentes (Navas, 2011; Acuña, 2011; Bocanegra y Díaz, 2013). Estas publicaciones tienen varias implicaciones para la diseminación de la $A C$ en Iberoamérica. Los resultados de estas revisiones, adaptaciones de escalas e intervenciones sugieren que la $A C$ es un tratamiento promisorio que se adapta a las condiciones culturales y particulares de nuestra población, aunque se nota una ausencia de publicaciones relacionadas con los posibles trabajos que se estén realizando en Iberoamérica (en especial las tesis en Colombia que aún no están publicadas y la traducción de la guía BATD-r en Argentina que está en proceso de publicación). Las recomendaciones de estas investigaciones redundan en la necesidad de contemplar variables culturales como el familismo (Jiménez, Bernal y Roselló, 2009) y consideraciones especiales para algunos grupos etarios.

Las direcciones futuras de la $A C$ están relacionadas con continuar consolidando la evidencia empírica de su eficacia 
tanto en población clínica como en población no clínica; realizar estudios en Latinoamérica que muestren que es igualmente aplicable a dicha población; realizar adaptaciones, validaciones y estandarizaciones de las escalas desarrolladas para el modelo (v.gr. EROS y BADS) en población iberoamericana; compilar más información vinculada con el entrenamiento a profesionales y no profesionales para la aplicación de la activación simple; consolidar protocolos o guías de atención de una sola sesión para población no clínica, especialmente en poblaciones educativas $u$ organizacionales donde se hayan detectado indicadores depresivos en procesos de selección, asesoría académica o consejería; desarrollar formatos y registros para aplicaciones de smartphones que faciliten su diligenciamiento y accesibilidad para poblaciones que disponen de este tipo de recursos tecnológicos, tales como Cognitive Diary CBT Self-Help, Emotios Diary CBT Basic y CBT Logger, entre otros disponibles para terapia cognitivo conductual; explorar recursos como los videojuegos para el tratamiento con población adolescente, por ejemplo el SPARX, juego de fantasía interactivo diseñado para atender depresión en adolescentes desde un modelo cognitivo-conductual (Merry et al., 2012).

\section{Referencias}

Acuña, L. (2011). Efectos de la aplicación de la terapia de activación conductual sobre la actividad fisica y la percepción de satisfacción en tres pacientes con enfermedad renal crónica terminal (tesis no publicada). Universidad Javeriana

Aguado, H., Silberman, R. y Manrique, E. (2005). Lineamientos para la Evaluación y Conceptualización Cognitivo- Conductual de la Depresión. Revista de Psiquiatría y Salud Mental Hermilio Valdizan, 6, 63-64.

Armento, M., McNulty, J. y Hopko, D. (2012). Behavioral Activation of Religious Behaviors (BARB): Randomized Trial With Depressed College Students. Psychology of Religion and Spirituality, 4, 206-222. Doi: $10.1037 / \mathrm{a} 0026405$.

Bailey, D. y Arco, L. (2010). Effects of a Brief Behavioural Activation Treatment on Activities of Various Difficulty and Depression. Behaviour Change, 27, 184-197.

Bajona, C. (2012). Terapia de grupo cognitivo-conductual con activación conductual para la depresión. ISEP Science, 2, 20-34.

Barraca, J. (2005). La Mente o la Vida: Una aproximación a la Terapia de Aceptación y Compromiso. Bilbao: Ed. Desclée de Brouwer, colección Serendipity.

Barraca, J. (2009). La Activación Conductual (AC) y la Terapia de Activación Conductual para la depresión (TACD). Dos protocolos de tratamiento desde el modelo de la Activación Conductual. Edu Psykhé. Revista de Psicología y Pedagogía, 8, 23-50.

Barraca, J. (2010). Tratamiento de activación conductual para la depresión (TACD): Descripción, técnicas y aplicación. Prolepsis, Colegio Oficial de Psicólogos de Castilla y León, 5, 27-39.

Barraca, J. (2011a). ¿Aceptación o control mental? Terapias de aceptación y mindfulness frente a las técnicas cognitivo-conductuales para la eliminación de pensamientos intrusos. Análisis y Modificación de Conducta, 37, 43-63.
Barraca, J. (2011b). Behavioral Activation Intervention in a Patient With Depressive Symtomatology. Psicología en España, 15, 22-32.

Barraca, J. y Pérez, M. (2010). Adaptación Española del Environmental Reward Observation Scale (Eros). Ansiedad y Estrés. 16, 95-107.

Barraca, J., Pérez, M. y Lozano, J. (2011). Avoidance and Activation as Keys to Depression: Adaptation of the Behavioral Activation for Depression Scale in a Spanish Sample. The Spanish Journal of Psychology, 14, online first.

Becerra, A., Lugo, I. y Reynoso, L. (2013). Terapia de Activación Conductual en Pacientes Oncológicos: Una Propuesta de Intervención. Psicooncología, 10, 163-168. Doi: 10.5209/rev PSIC.2013.v10.41969.

Beck, A. T., Rush, A. J., Shaw, B. F. y Emery, G. (1983). Terapia cognitiva de la depresión. Bilbao: Desclee de Brower.

Berenzon, S., Lara, M. A., Robles, R. y Medina, M. E. (2013). Depresión: estado del conocimiento y la necesidad de políticas públicas y planes de acción en México. Salud Pública de México, 55, 74-80.

Bocanegra, S. y Díaz, M. (2013). Aplicación del tratamiento de activación conductual breve para la depresión en adolescentes: estudio de caso único. Fundación Universitaria Konrad Lorenz. Tesis para optar por el título de maestría en psicología clínica (aún no publicada).

Bottonari, K., Roberts, J., Thomas, S. y Read, J. (2008). Stop Thinking and Start Doing: Switching From Cognitive Therapy to Behavioral Activation in a Case of Chronic Treatment-Resistant Depression. Elsevier, 8, 376-386.

Butler, A., Chapman, J., Forman, E. y Beck, A. (2006). The Empirical Status Of Cognitive-Behavioral Therapy: A Review Of Meta-Analyses. Elsevier. Clinical Psychology Review, 26, 17-31. Doi: 10.1016/j. cpr.2005.07.003.

Castro, L., Escobar, J. M., Sáenz, C., Delgado, L., Aparicio, S., Molano, J. C. y Noguera, E. (2012). Salud mental en el hospital general: resultados del Cuestionario de Salud del Paciente (PHQ) en cuatro servicios de atención. Revista Colombiana de Psiquiatría, 41, 61-85.

Caycedo, C., Sandoval, M., Gutiérrez, C. y Pereira, C. (1994). Ley de Igualación: Conceptos Básicos, Evolución y Perspectivas. Suma Psicológica, 1, 38-50.

Collado, A., Castillo, S., Maero, F., Lejuez, C. W. y MacPherson, L. (2014). Pilot of the brief behavioral activation treatment for depression in Latinos with limited English proficiency: Preliminary evaluation of efficacy and acceptability. Behavior Therapy, 45, 102-115.

Cuijpers, P., Van Straten, A. y Warmerdam, L. (2007). Behavioral activation treatments of depression: A meta-analysis. Clinical psychology review, 27, 318-326.

Cullen, J., Spates, R., Pagoto, S. y Doran (2006). Behavioral Activation Treatment for Major Depressive Disorder: A Pilot Investigation. The Behavior Analyst Today, 7, 151-166.

DeRubeis, R., Hollon, S., Amsterdam, J., Shelton, R., Young, P., Salomon, R., O'Reardon, J., Lovett, M., Gladis, M., Brown, L. y Gallop, R. (2005). Cognitive Therapy vs Medications in the Treatment of Moderate to Severe Depression. Arch Gen Psychiatry, 62, 409-416. Doi:10.1001/ archpsyc.62.4.409.

Díaz, N. (2012). Depresión y Factores Asociados en Estudiantes de la Universidad Nacional de Colombia (Doctoral dissertation). Universidad Nacional de Colombia.

Díaz, D. N. y Ruiz, A. (2002). Estudio epidemiológico de la población asistida en el Departamento de Psiquiatría del Hospital. Revista Médica Uruguaya, 18, 48-58.

Dimidjian, S., Martell, C., Addis, M. y Herman-Dunn, R. (2008). Behavioral Activation for Depression. En Barlow, D. (Ed.). Clinical Handbook of Psychological Disorders(pp. 328-364). New York: The Guilford Press.

Ekers, D., Godfrey, C., Gilbody, S., Parrott, S., Richards, D., Hammond, D. y Hayes, A. (2011). Cost utility of behavioural activation delivered by the non-specialist. The British Journal of Psychiatry. Doi:10.1192/ bjp.bp. 110.090266 .

Ekers, D. (2011). Behavioural Activation for Depression: a Systematic Review and Controlled Clinical Trial (thesis submitted for the degree of PhD). The Univesity of York. Health Sciences. 
Ekers, D., Richards, D. y Gilbody, S. (2008). A meta-analysis of randomized trials of behavioural treatment of depression. Psychological Medicine, 38, 611-623. Doi: 10.1017/S0033291707001614.

Fernández, C., Villoria, E., Amigo, I., Padierna, C., Gracia, J., Fernández, R. y Peláez, I. (2011). Terapia de activación conductual en pacientes con cáncer. Anales de Psicología, 7, 278-291.

Freij, K. y Masri, N. (2008). The Brief Behavioral Activation Treatment for Depression - A psychiatric pilot study. Nordic Psychology, 60, 129140. Doi: 10.1027/1901-2276.60.2.129.

Gawrysiak, M., Nicholas, C. y Hopko, D. (2009). Behavioral Activation for Moderately Depressed University Students: Randomized Controlled Trial. Journal of Counseling Psychology, 56, 468-475.

Gawrysiak, M., Carvalho, J., Rogers, B., Nicholas, C., Dougherty, J. y Hopko, D. (2012). Neural Changes following Behavioral Activation for a Depressed Breast Cancer Patient: A Functional MRI Case Study. Case Reports in Psychiatry. ID 152916, 1-8. Doi:10.1155/2012/152916.

Gotlib, I. H. y Hammen, C. L. (2009). Introduction. En I.H. Gotlib y C.L. Hammen (Eds.). Handbook of depression (pp. 1-20). Nueva York: Guilford.

Gómez, C., Bohórquez, A., Pinto, D., Gil, J., Rondón, M. y Díaz-Granados, N. (2004). Prevalencia de depresión y factores asociados con ella en la población colombiana. Revista Panamericana de Salud Pública, 16, 378-86.

Hollon, S. y Ponniah, K. (2010). A Review Of Empirically Supported Psychological Therapies For Mood Disorders In Adults. Depression and Anxiety, 27, 891-932.

Hopko, D., Armento, M., Hunt, M., Bell, J. y Lejuez, C. (2005). Behavior therapy for depressed cancer patients in primary care. Psychotherapy: Theory, Research, Practice, Training Copyright 2005 by the Educational Publishing Foundation, 42, 236-243.

Hopko, D., Armento, M., Robertson, S., Ryba, M., Carvalho, J., Colman, L., Mullane, C., Gawrysiak, M., McNulty, J., Bell, J. y Lejuez, C. (2011). Brief Behavioral Activation and Problem-Solving Therapy for Depressed Breast Cancer Patients: Randomized Trial. Journal of Consulting and Clinical Psychology, 79, 834-849. Doi: 10.1037/a0025450.

Hopko, D. R., Lejuez, C. W., Ruggiero, K. J. y Eifert, G. H. (2003). Contemporary behavioral activation treatments for depression: Procedures, principles, and progress. Clinical Psychology Review, 23, 699-617.

Hopko, D. R., Lejuez, C. W. y Hopko, S. D. (2004). Behavioral activation as an intervention for coexistent depressive and anxiety symptoms. Clinical Case Studies, 3, 37-48.

Jacob, M., Keeley, M., Ritschel, L. y Craighead, E. (2011). Behavioral activation for the treatment of low-income, African American adolescents with major depressive disorder: a case series. Clinical Psychology and Psychotherapy, 20, 87-96. Doi: 10.1002/cpp.774

Jacobson, N., Dobson, K., Truax, P., Addis, M., Koerner, K., Gollan, J., Gortner, E. y Prince, S. (1996). A Component Analysis of CognitiveBehavioral Treatment for Depression. Journal of Consulting and Clinical Psychology. 64, 295-304.

Jiménez, M., Bernal, G. y Roselló, J. (2009). Clinical case study: CBT for depression in a Puerto Rican Adolescent: Challenges and variability in treatment response. Depression and Anxiety, 26, 98-103. Doi: 10.1002/da.20457.

Kanter, J., Busch, A., Weeks, C. y Landes, S. (2008). The Nature of Clinical Depression: Symptoms, Syndromes, and Behavior Analysis. The Behavior Analyst, 31, 1-21.

Kanter, J., Busch, A. y Rusch, L. (2009). Behavioral Activation: Distinctive Features. New York: Routledge/Taylor y Francis e-Library.

Kanter, J., Diéguez, G., Rusch, L., Busch, A. y Santiago-Rivera, A. (2008). Behavioral Activation for Latinos With Depression. Clinical Case Studies, 7, 491-506.

Kanter, J., Santiago-Rivera, A., Rusch, L., Busch, A. y West, P. (2010). Initial outcomes of a culturally adapted behavioral activation for Latinas diagnosed with depression at a community clinic. Behavior Modification, 34, 120-144.

Kanter, J., Mulick, P., Busch, A., Berlin, K. y Martell, C. (2006). The Behavioral Activation for Depression Scale (BADS): Psychometric
Properties and Factor Structure. J Psychopathol Behav Assess. Journal of Psychopathology and Behavioral Assessment. Doi: 10.1007/ s10862-006-9038-5.

Kessler, R. y Wang, P. (2009). Epidemiology of Depression. En Gotlib, I. y Hammen, C. (Eds.). Handbook of Depression (pp. 5-22). New York: The Gilford Press.

Kohn, R., Levav I., Caldas de Almeida, J., Vicente, B., Andrade, L., Caraveo-Anduaga, J., Saxena, S. y Saraceno, B. (2005). Los trastornos mentales en América Latina y el Caribe: asunto prioritario para la salud pública. Revista Panamericana de Salud Pública, 18, 229-40.

Lejuez, C. W., Hopko, D. R., LePage, J., Hopko, S. D. y McNeil, D. W. (2001). A brief behavioral activation treatment for depression: A Randomized Pilot Trial Within an Inpatient Psychiatric Hospital. Cognitive and Behavioral Practice, 8, 164-175. Doi: 10.1177/0145445503255489.

LeJuez, C., Hopko, D., Acierno, R., Daughters, S. y Pagoto, S. (2010). Ten Year Revision of the Brief Behavioral Activation Treatment for Depression (BATD): Revised Treatment Manual (BATD-R). Department of Psychology.

Center for Addictions, Personality, and Emotion Research (CAPER). University of Maryland. College Park, MD 20742. Recuperado desde http://web.utk.edu/ dhopko/BBATD2010_BMOD.pdf.

Lewinsohn, P., Hoberman, H. y Clarke, G. (1989). The Coping With Depression Course: Review and future directions. Canadian Journal of Behavioural Science/Revue canadienne des sciences du comportement, 21, 470-493. Doi: 10.1037/h0079846.

Lewinsohn, P. (1975). Engagement in Pleasant Activities and Depression Level. Journal ol Abnormal Psychology, 84(6), 729-731. Doi: 10.1037/0021-843X.84.6.729.

Lezama, S. (2012). Propiedades Psicométricas de la Escala de Zung para Síntomas Depresivos en Población Adolescente Escolarizada Colombiana.

López, M., Montoya, D. y Dussán, C. (2012). Caracterización de los asistentes al centro de atención psicológica de la Universidad de Manizales, 2006-2010. Hacia la Promoción de la Salud. Nombre revista, 17, 149-166.

MacPherson, L., Tull, M. T., Matusiewicz, A. K., Rodman, S., Strong, D. R., Kahler, C. W. y Lejuez, C. W. (2010). Randomized controlled trial of behavioral activation smoking cessation treatment for smokers with elevated depressive symptoms. Journal of consulting and clinical psychology, 78, 55.

Magidson, J., Gorka, S., MacPherson, L., Hopko, D., Blanco, C., Lejuez, C.W. y Daughters, S. (2011). Examining the effect of the Life Enhancement Treatment for Substance Use (LETS ACT) on residential substance abuse treatment retention. Addictive Behaviors, 36, 615-623.

Martell, C., Addis, M. y Jacobson, N. (2001). Depression in Context: Strategies for Guided Action. Norton y Co, New York.

Martell, C., Dimidjian, S. y Dunn, R. (2010). Behavioral Activation for Depression: A Clinician's Guide. New York. The Guilford Press.

Mazzucchelli, T., Kane, R. y Rees, C. (2009). Behavioral Activation Treatments for depression in Adults: A Meta-analysis and Review Clinical Psychology. Science and Practice. 16, 383-411. Doi:10.1016/j. cpr.2006.11.001.

Merry, S., Stasiak, K., Shepherd, M., Frampton, C., Fleming, T. y Lucassen, M. (2012). The effectiveness of SPARX, a computerised self help intervention for adolescents seeking help for depression: randomised controlled non-inferiority trial. British Medical Journal. 344, 598. Doi: 10.1136/bmj.e2598.

Minoletti, A. y Zaccaria, A. (2005). Plan Nacional de Salud Mental en Chile: 10 años de experiencia. Revista Panamericana de Salud Pública, 18, 346-358.

Navas, M. S. (2011). Efecto de la terapia de activación conductual en las conductas depresivas de tres consultantes (tesis no publicada). Universidad Javeriana.

Ortiz, L., López, S. y Borges, G. (2007). Desigualdad socioeconómica y salud mental: revisión de la literatura latinoamericana. Socioeconomic inequality and mental health: a Latin American literature review. $\mathrm{Ca}$ dernos de saúde pública, 23, 1255-1272. 
Pérez, M. (1996). La psicoterapia desde el punto de vista conductista. Biblioteca nueva.

Pérez, M. (2007). La Activación Conductual y la desmedicalización de la Depresión. Papeles del Psicólogo, 28, 97-110.

Pérez, M. (2008). Desenredamiento auto-reflexivo y activación conductual: claves para la terapia. Prolepsis, 0, 17-43.

Pérez, M. (2012). Third-Generation Therapies: Achievements and challenges. International Journal of Clinical and Health Psychology, 12, 291-310.

Posada-Villa, J. A., Aguilar-Gaxiola, S., Magaña, C. G. y Gómez, L. C. (2004). Prevalencia de trastornos mentales y uso de servicios: resultados preliminares del Estudio Nacional de Salud Mental, Colombia, 2003. Revista Colombiana de Psiquiatría, 33, 241-62.

Puspitasari, A., Kanter, J., Koerner, K., Murphy, J. y Crowe, A. (2013). Developing an Online, Modular, Active Learning Training Program for Behavioral Activation. Psychotherapy, 50, 256-265. Doi: 10.1037/ a0030058.

Rehm, L. (1978). Mood, Pleasant Events, and Unpleasant Events: Two Pilot Studies. Journal of Consulting and Clinical Psychology, 46, 854859. Doi: 10.1037/0022-006X.46.5.854.

Rodríguez, M. R., Rincón, H. H. G., Velasco, P. M. M., Hernández, A. C. A., Ramos, M. L. y González, J. A. (2012). Prevalencia de trastornos mentales en una población que demanda servicios de salud en una
IPS de baja complejidad y su asociación con la ideación suicida y la discapacidad percibida. Revista Facultad Nacional de Salud Pública, 30, 141-151.

Romero, R., Oñate, S. y Vucinovich, N. (2011). Terapia de activación conductual para la depresión: aplicación a un paciente con esquizofrenia paranoide. Análisis y Modificación de Conducta, 37, 65-75.

Roses, M. (2005). La salud mental: una prioridad de salud pública en las Américas. Revista Panamericana de Salud Pública, 18, 223-225.

Ruggiero, K., Morris, T., Hopko, D. y Lejuez, C. W. (2007). Application of Behavioral Activation Treatment for Depression to an Adolescent with a History of Child Maltreatment. Clinical Case Studies, 2, 1-17. Doi: $10.1177 / 1534650105275986$.

Salinas, J. (2010). El Tratamiento de la depresión con un enfoque de Activación Conductual: Un Caso. Revista Electrónica de Psicología Iztacala, 13, 3.

Santiago-Rivera, A., Kanter, J., Benson, G., Derose, T., Illes, R. y Reyes, W. (2008). Behavioral Activation As An Alternative Treatment Approach For Latinos With Depression. Psychotherapy Theory, Research, Practice, Training, 45, 173-185. Doi: 10.1037/0033-3204.45.2.173.

Spates, R., Pagoto, S. y Kalata, A. (2006). A Qualitative And Quantitative Review of Behavioral Activation Treatment of Major Depressive Disorder. The Behavior Analyst Today, 7, 508-521.

World Health Organization (2008). The global burden of disease: 2004 update. Geneva, Switzerland: World Health Organization. 
